\title{
Hidden costs of control: four repetitions and an extension
}

\author{
Anthony Ziegelmeyer • Katrin Schmelz • \\ Matteo Ploner
}

\begin{abstract}
We report four repetitions of Falk and Kosfeld's (Am. Econ. Rev. 96(5):1611-1630, 2006) low and medium control treatments with 476 subjects. Each repetition employs a sample drawn from a standard subject pool of students and demographics vary across samples. We largely confirm the existence of hidden costs of control but, contrary to the original study, hidden costs of control are usually not substantial enough to significantly undermine the effectiveness of economic incentives. Our subjects were asked, at the end of the experimental session, to complete a questionnaire in which they had to state their work motivation in hypothetical scenarios. Our questionnaires are identical to the ones administered in Falk and Kosfeld's (Am. Econ. Rev. 96(5):1611-1630, 2006) questionnaire study. In contrast to the game play data, our questionnaire data are similar to those of the original questionnaire study. In an attempt to solve this puzzle, we report an extension with 228 subjects where performance-contingent earnings are absent i.e. both principals and agents are paid
\end{abstract}

The order of authors reflects their relative contributions to the article.

\author{
A. Ziegelmeyer \\ Strategic Interaction Group, Max Planck Institute of Economics, Kahlaische Strasse 10, 07745 Jena, \\ Germany \\ e-mail: ziegelmeyer@econ.mpg.de \\ K. Schmelz ( $\varangle)$ \\ Thurgau Institute of Economics, University of Konstanz, Hauptstrasse 90, 8280 Kreuzlingen 2, \\ Switzerland \\ e-mail: schmelz@twi-kreuzlingen.ch \\ M. Ploner \\ Department of Economics, Computable and Experimental Economics Laboratory, University of \\ Trento, Via Inama 5, 38100 Trento, Italy \\ e-mail: matteo.ploner@unitn.it
}


according to a flat participation fee. We observe that hidden costs significantly outweigh benefits of control under hypothetical incentives.

Keywords Control $\cdot$ Laboratory experiments $\cdot$ Motivation · Principal-agent theory

JEL Classification C81 C $91 \cdot$ M52

\section{Introduction}

Standard principal-agent theory endorses the view that tighter monitoring invariably leads to profitable increases in agents' work effort and recommends principals to offer jobs characterized by limited effort discretion. However, since the work by Deci (1971), many social scientists reject this view and acknowledge that monitoring is likely to have two opposite effects on the agents' performance: A disciplining effect (monitoring limits shirking and therefore raises work effort) and a crowding-out effect (monitoring undermines intrinsic motivation and therefore reduces work effort). Whether monitoring agents is beneficial to the principal depends on the relative size of the two countervailing effects, and a worthwhile goal for economic research is to identify the conditions under which crowding-out effects are predicted to dominate disciplining effects.

Frey (1993) distinguishes between principal-agent relationships and he reasons that the disciplining effect is likely to dominate when the relationship is abstract, as in competitive markets, while the crowding-out effect is likely to dominate in more personalized relationships. This proposition is supported by the field evidence in Barkema (1995) and by a large body of evidence in psychology which indicates that distant principal-agent relationships are often characterized by a controlling leadership style whereas agents' autonomy is more prominent in close principal-agent relationships (Antonakis and Atwater 2002; Stanton 2000 and the references therein). Recent experimental evidence collected by economists provides additional support for Frey's proposition. For example, Dickinson and Villeval (2008) establish that a personal relationship between principal and agent is a major condition for a substantial crowding-out effect to emerge, and Bartling et al. (2011) show that offering discretion to an anonymous agent is not profitable on its own but that the combined strategy of offering discretion, paying high wages, and screening based on past efforts is profitable.

Contrary to this large body of evidence, Falk and Kosfeld (2006)—henceforth F\&K-show experimentally that motivation crowding-out effects are substantial enough to significantly undermine the effectiveness of control in abstract relationships and in the absence of complementarities between effort discretion and other elements of the employment strategy. In their main treatments, F\&K implement a principal-agent game where the principal either restricts the agent's choices by setting $x_{\min }=\underline{x}$ or leaves the agent's choices unrestricted by setting $x_{\min }=0$ and the agent then chooses a transfer $x \in\left\{x_{\min }, x_{\min }+1, \ldots, 119,120\right\}$ resulting in a (monetary) payoff of $2 x$ and $120-x$ experimental currency units (ECUs) for the principal and the agent respectively. The minimum transfer requirement $\underline{x} \in\{5,10,20\}$ is a treatment 
variable which corresponds to the principal's degree of control. Experimental results show that control entails hidden costs caused by the existence of agents who choose a lower transfer if controlled than otherwise. Most strikingly, hidden costs outweigh the benefits of control in the low and medium control treatments (where $\underline{x}$ equals 5 and 10 respectively) which implies that principals earn less when they control their agents than when they do not control them. If robust, F\&K's experimental results have far-reaching implications for the design of employment contracts. Monitoring strategies would not only have to regulate the margin of employees who exploit their freedom to shirk but they should also sustain the motivation of the large fraction of employees disinclined to shirk.

In this paper, we first report four repetitions of F\&K's low and medium control treatments. Our repetitions largely reproduce the conditions of the original study with different subject pools (like F\&K each of our experiments employs a standard subject pool of students), and either a show-up fee of 25 ECUs (first three repetitions) or the same 50 ECUs show-up fee as F\&K (fourth repetition). The primary purpose of our repetitions is to check the robustness of F\&K's experimental results to small changes in subject pools. Except in our second repetition, we confirm the existence of statistically significant hidden costs of control. More importantly, and in contrast to F\&K's findings, hidden costs almost never statistically significantly outweigh benefits of control in our four repetitions.

Once game play data had been collected, subjects were asked to complete a questionnaire in which they had to state their work motivation in hypothetical scenarios. Our questionnaires are identical to the ones F\&K administered in their questionnaire study. In contrast to the game play data, our questionnaire data are similar to F\&K's questionnaire data. Like in the original questionnaire study, we observe that in each scenario stated work motivation is statistically significantly lower in the condition where the principal controls or uses explicit incentive devices than in the condition where the principal trusts the agent. We decided to investigate the influence of hypothetical incentives on the play of the principal-agent game. In the second part of the paper, we report an extension of F\&K's low and medium control treatments where performance-contingent earnings are absent i.e. both principals and agents are paid according to a flat participation fee. We observe that hidden costs statistically significantly outweigh benefits of control which suggests that the magnitude of hidden costs of control is larger under hypothetical than under real incentives.

The remainder of this paper is organized as follows. Section 2 summarizes F\&K's experimental findings in their main treatments. Section 3 outlines the experimental design and procedures of our study. Section 4 describes our results. Section 5 concludes.

\section{Falk \& Kosfeld's main treatments and hidden costs of control}

F\&K's main treatments consist of a low $(\underline{x}=5)$, a medium $(\underline{x}=10)$, and a high $(\underline{x}=20)$ control treatment (referred to as C5, C10, and C20 respectively). Principals and agents interact only once and F\&K make use of the strategy method to elicit agents' transfers. Concretely, each agent is asked to choose a pair of transfers 
$\left(x^{C}, x^{N C}\right)$ where $x^{C} \in\{\underline{x}, \underline{x}+1, \ldots, 120\}$ is payoff-relevant in case the principal imposes a compulsory transfer and $x^{N C} \in\{0,1, \ldots, 120\}$ is payoff-relevant in case the principal does not impose a compulsory transfer. A total of 140, 144, and 134 subjects participated in the control treatments $\mathrm{C} 5, \mathrm{C} 10$, and $\mathrm{C} 20$ respectively.

For a given agent's pair of transfers $\left(x^{C}, x^{N C}\right)$, the decision to impose a compulsory transfer has a total effect on the principal's payoff which is given by $2\left(x^{C}-x^{N C}\right)$. This total effect can be expressed as the sum of the direct and indirect effects of control which are given by $2 \max \left\{\underline{x}-x^{N C}, 0\right\}$ and $2\left(x^{C}-\max \left\{\underline{x}, x^{N C}\right\}\right)$ respectively (Schnedler and Vadovic 2011). The indirect effect of control is positive if either $x^{C}>\underline{x}>x^{N C}$ or $x^{C}>x^{N C} \geq \underline{x}$, in which case there are hidden benefits of control. The indirect effect is negative if $x^{N C}>x^{C}$, in which case there are hidden costs of control.

F\&K's key findings can be summarized as follows. First, statistically significant hidden costs of control are observed in all control treatments (C5, C10, and C20). Second, most agents choose $x^{N C}>x^{C}$ in $\mathrm{C} 5$ and C10. Almost identical proportions of agents exhibit positive and negative reactions to control in $\mathrm{C} 20$. Third, the average transfer by agents is higher when agents are not controlled than when they are $\left(\bar{x}^{C}-\right.$ $\left.\bar{x}^{N C}<0\right)$ i.e. hidden costs of control outweigh the benefits. These differences are statistically significant in $\mathrm{C} 5$ and $\mathrm{C} 10$ but not in C20. Fourth, principals anticipate the adverse effect of control and choose to leave transfers unrestricted in $\mathrm{C} 5$ and $\mathrm{C} 10$. About half of the principals choose to leave transfers unrestricted in C20.

As $\mathrm{F} \& \mathrm{~K}$ explain in their paper, profit-maximizing principals will force agents to transfer at least $\underline{x}$ ECUs if all agents are profit-maximizers, and also if some agents have a concern for equity and/or efficiency. Even some applications based on psychological games rule out the possibility that, in equilibrium, the principal leaves transfers unrestricted and the agent responds positively to the principal's signal of kindness (Rabin 1993; Dufwenberg and Kirchsteiger 2004; Falk and Fischbacher 2006). Intention-based reciprocity might explain the crowding-out effect if the propensity for reciprocity varies in the population and propensities are private information (von Siemens 2011). Signaling motives also have the potential to rationalize agents' negative reactions to control (Sliwka 2007; Ellingsen and Johannesson 2008).

\section{Design and procedures}

Our study consists of five experiments. Experiments 1, 2, 3 and 5 are repetitions of F\&K's low and medium control treatments. ${ }^{1}$ Our repetitions largely reproduce the original conditions with different subject pools and either a show-up fee of 25 ECUs (Experiments 1, 2 and 3) or the same 50 ECUs show-up fee as F\&K (Experiment 5). In each repetition, after having played the principal-agent game, subjects stated their work motivation in hypothetical scenarios identical to the ones F\&K administered in their questionnaire study. The evidence collected in Experiments 1,2 and 3 led us to conjecture that the magnitude of hidden costs of control is larger under hypothetical

\footnotetext{
${ }^{1}$ Since the striking nature of F\&K's experimental findings is confined to the low and medium control treatments, a robustness check of the results in the high control treatment seems of little interest.
} 
than under real incentives. Experiment 4 tests this conjecture by asking agents and principals to make hypothetical choices for a flat fee in the low and medium control treatments. Experiment 5 investigates whether the difference between F\&K's results and the results of Experiments 1,2 and 3 originates from the relative size of the show-up payment with respect to contingent rewards.

\subsection{The four repetitions}

In Experiment 1, subjects are students from the University of Jena (federal state of Thuringia, Germany) and the sample composition according to educational background is (almost) equally distributed among the primary fields of academic study.

In Experiment 2, subjects are students from the University of Trento (province of Trento, Italy) and about $60 \%$ of the sample was majoring in business administration or economics. ${ }^{2}$ Experiment 2 allows us to further assess the robustness of F\&K's experimental results by rerunning Experiment 1 on a new pool of subjects and it partly addresses the potential issue of cross-regional differences (Jena and Trento are two cities of comparable size). To control for potential experimental artifacts, we followed Roth et al. (1991) on appropriate designs for cross-country experiments. In particular, we did our best to control for experimenter and language effects (see Sect. 3.3). ${ }^{3}$

As shown below, the experimental results of our two first repetitions differ. This difference cannot be attributed solely to cross-regional differences in subjects' attitudes since they confound with sample differences in educational background. The majority of subjects in Experiment 2 are business and economics majors while such students represent less than $30 \%$ of Experiment 1's sample. To assess the severity of the confound, we decided to run Experiment 3 with students from the University of Jena and a sample composition according to educational background identical to Experiment 2.

In Experiments 1, 2 and 3, our subjects received a show-up fee of 25 ECUs (2.50 Euro) which is half the show-up fee F\&K paid to their subjects. We decided to deviate from the original procedures because subjects (almost) systematically receive a 2.50 Euro show-up fee at the laboratories in Jena and Trento. Accordingly, we place more emphasis on performance-contingent earnings in Experiments 1,2 and 3 compared to F\&K's low and medium control treatments. An anonymous referee urged us to conduct an additional repetition with the same show-up payment as F\&K. Experiment 5 employs a similar sample as Experiment 1 but subjects received a show-up fee of 50 ECUs. $^{4}$

\footnotetext{
${ }^{2}$ At the time of the experiment, this composition of the sample was not unusual in Trento since students from other majors were less aware of the possibility to participate in economic experiments, and the laboratory is located near the faculty of economics.

${ }^{3}$ The currency effect is of no concern since both Germany and Italy belong to the Euro zone and the price levels in the two countries are equivalent. According to Eurostat, the statistical office of the European Union, the 2007 comparative price level indices equal 101.7 and 102.5 in Germany and Italy respectively. In Switzerland, the 2007 price level index equals 125.1 and it has been about 140 in the period during which F\&K conducted their experiment.

${ }^{4}$ It remains unclear whether Experiment 5 with an uncommon show-up fee of 5 Euro is more comparable to F\&K's low and medium control treatments than Experiment 1. Though we did our best to reproduce the
} 


\subsection{The extension}

Experiment 4 is an extension of F\&K's C5 and C10 treatments where performancecontingent earnings are absent i.e. both principals and agents are paid a flat participation fee for completing the experiment. Subjects are students from the University of Jena and the sample composition according to educational background is identical to the one in Experiment 1.

In each of the five panels of Table 1 the 6th row shows for each experiment the sample composition according to educational background. In Experiments 1, 4 and 5 , the list of four integers in each column corresponds to the percentages of subjects whose academic major belongs to: (i) Business administration \& economics; (ii) Other behavioral \& social sciences (education sciences, law, political science, psychology, and sociology); (iii) Humanities; and (iv) Engineering, life \& natural sciences. In Experiments 2 and 3, the category 'other behavioral \& social sciences' consists mainly of law students and therefore the percentage of law students is indicated separately by the first element of the sum.

\subsection{Practical procedures}

As in F\&K, all experiments were computerized with the help of the software z-Tree (Fischbacher 2007). We used a slightly modified version of the original code (we enlarged the fonts to facilitate subjects' reading of the computer screens and we added F\&K's questionnaire on work motivation). All sessions of Experiments 1, 3, 4 and 5 were conducted at the Experimental Laboratory of the Max Planck Institute of Economics (ELMPIE) in Jena. Subjects were invited using the ORSEE recruitment system (Greiner 2004). Almost all the 588 subjects were students from the Friedrich Schiller University of Jena and a few (less than 10\%) were students from the University of Applied Sciences Jena. The six sessions of Experiment 2 were conducted at the Computable and Experimental Economics Laboratory (CEEL) of the University of Trento. All 116 subjects were students from the University of Trento. ${ }^{5}$ No subject participated in more than one session. Most subjects had participated in earlier economics experiments, but all were inexperienced in the sense that they had never taken part in an earlier session of this type. Subjects interacted only once and each session lasted on average 40 minutes. Table 1 summarizes the experimental conditions of our study. Note that gender composition is approximately balanced not only in each experiment but in the large majority of sessions. ${ }^{6}$

original conditions, minor variations in the procedures or in the subject pool are unavoidable. We believe that genuine experimental regularities should be robust to such minor variations. Admittedly, variations in the subject pool could be large as we were unable to obtain any information concerning the distribution of demographics in F\&K's sample.

${ }^{5}$ Experiments 1 and 2 (respectively 3,4 and 5) were conducted under the supervision of the first author with the help of the third (respectively second) author.

${ }^{6}$ Compared to Experiment 1, two additional sessions had to be conducted in Experiment 2 in order to collect the same number of observations since the CEEL has a capacity of 20 participants whereas the ELMPIE has a capacity of 32 participants. The sessions of Experiment 3 took place at the end of the term break which led to many no-shows and forced us to conduct a total of 8 sessions. Finally, since hypothetical incentives usually increase the "noise" in subjects" decisions, we collected twice the amount of data in Experiment 4 compared to Experiment 1. 
Table 1 Experimental conditions

\begin{tabular}{|c|c|c|c|c|}
\hline \multirow{9}{*}{$\begin{array}{c}\text { Experiment } \\
1\end{array}$} & & $\mathrm{C} 5$ & $\mathrm{C} 10$ & Pooled \\
\hline & Location & Jena & & \\
\hline & Number of sessions & 2 & 2 & 4 \\
\hline & Number of subjects & 60 & 58 & 118 \\
\hline & Gender ( $\%$ female) & 50.00 & 53.12 & 51.56 \\
\hline & Average age (std. dev.) & $24.20(2.35)$ & $24.10(2.05)$ & $24.15(2.19)$ \\
\hline & Educational background (\%) & $27 / 22 / 20 / 31$ & $31 / 20 / 25 / 19$ & $29 / 21 / 23 / 25$ \\
\hline & Agents' average earnings & $10.50(1.31)$ & $10.52(1.07)$ & $10.51(1.19)$ \\
\hline & Principals' average earnings & $3.00(2.63)$ & $2.97(2.13)$ & $2.98(2.38)$ \\
\hline \multirow{8}{*}{$\begin{array}{c}\text { Experiment } \\
2\end{array}$} & Location & Trento & & \\
\hline & Number of sessions & 3 & 3 & 6 \\
\hline & Number of subjects & 56 & 60 & 116 \\
\hline & Gender ( $\%$ female) & 51.79 & 50.00 & 50.86 \\
\hline & Average age (std. dev.) & $21.21(2.34)$ & $21.75(2.18)$ & $21.49(2.27)$ \\
\hline & Educational background (\%) & $50 / 29+5 / 7 / 9$ & $63 / 17+5 / 8 / 7$ & $57 / 22+5 / 8 / 8$ \\
\hline & Agents' average earnings & $10.83(1.33)$ & $10.91(0.70)$ & $10.87(1.04)$ \\
\hline & Principals' average earnings & $2.34(2.67)$ & $2.19(1.40)$ & $2.26(2.09)$ \\
\hline \multirow{8}{*}{$\begin{array}{c}\text { Experiment } \\
3\end{array}$} & Location & Jena & & \\
\hline & Number of sessions & 4 & 4 & 8 \\
\hline & Number of subjects & 64 & 66 & 130 \\
\hline & Gender ( $\%$ female) & 51.56 & 50.00 & 50.77 \\
\hline & Average age (std. dev.) & $23.97(2.87)$ & $23.76(2.63)$ & $23.86(2.74)$ \\
\hline & Educational background (\%) & $60 / 22+9 / 6 / 3$ & $64 / 12+9 / 8 / 7$ & $62 / 17+9 / 7 / 5$ \\
\hline & Agents' average earnings & $10.08(1.97)$ & $10.53(1.12)$ & $10.31(1.60)$ \\
\hline & Principals' average earnings & $3.83(3.95)$ & $2.94(2.25)$ & $3.38(3.20)$ \\
\hline \multirow{8}{*}{$\begin{array}{c}\text { Experiment } \\
4\end{array}$} & Location & Jena & & \\
\hline & Number of sessions & 5 & 5 & 10 \\
\hline & Number of subjects & 114 & 114 & 228 \\
\hline & Gender ( $\%$ female) & 48.25 & 50.88 & 49.56 \\
\hline & Average age (std. dev.) & $24.71(2.90)$ & $24.13(2.89)$ & $24.42(2.90)$ \\
\hline & Educational background (\%) & $20 / 26 / 32 / 22$ & $25 / 32 / 20 / 23$ & $22 / 30 / 26 / 22$ \\
\hline & Agents' average earnings & $10.00(0.00)$ & $10.00(0.00)$ & $10.00(0.00)$ \\
\hline & Principals' average earnings & $10.00(0.00)$ & $10.00(0.00)$ & $10.00(0.00)$ \\
\hline \multirow{8}{*}{$\begin{array}{c}\text { Experiment } \\
5\end{array}$} & Location & Jena & & \\
\hline & Number of sessions & 2 & 2 & 4 \\
\hline & Number of subjects & 56 & 56 & 112 \\
\hline & Gender ( $\%$ female $)$ & 50.00 & 53.57 & 51.79 \\
\hline & Average age (std. dev.) & $24.04(2.90)$ & $24.20(2.40)$ & $24.12(2.65)$ \\
\hline & Educational background (\%) & $25 / 21 / 27 / 27$ & $27 / 21 / 23 / 29$ & $26 / 21 / 25 / 28$ \\
\hline & Agents' average earnings & $10.59(1.39)$ & $10.45(1.31)$ & $10.52(1.34)$ \\
\hline & Principals' average earnings & $2.81(2.79)$ & $3.11(2.62)$ & $2.96(2.68)$ \\
\hline
\end{tabular}

Notes: In Experiment 1, subjects' educational background has not been elicited directly. The percentages indicated in the table have been computed with the help of the lists of registered subjects generated by the online recruitment system. In treatment $\mathrm{C} 10$, the educational background of three registered subjects is missing.

Earnings are stated in Euro net of the show-up fee with standard deviations in parentheses. There was no show-up fee in Experiment 4 
At the beginning of each session subjects randomly drew a cubicle number. Half of the subjects were assigned the role of principal and the other half the role of agent, depending on their cubicle number. Cubicles were visually isolated from each other and communication between the subjects was strictly prohibited. Once all subjects sat down in their cubicles, instructions were distributed. Principals and agents were given different sets of instructions. In Experiments 1, 3 and 5 we used the exact same instructions as F\&K but modified them slightly to account for the change of currency, funding institution, and show-up fee in Experiments 1 and 3. For Experiment 2, the German instructions of Experiment 1 were translated into Italian, back-translated and compared to the original instructions by the third author and a professional translator until the rules of the experiment were explained as similarly as possible in both languages. The German instructions of Experiment 4 contain two additional paragraphs which explain that decisions are hypothetical and that each subject is paid a fixed amount of 10 Euro for participation (see Appendices A and B of the electronic supplementary material).

Each subject first read his own instructions silently and then, to ensure common information, a monitor read the common parts of the two sets of instructions aloud (the monitor was a native speaker who did not know the purpose of the experiment). Questions were answered privately. Like in F\&K's low and medium control treatments, understanding of the payoff functions was assured by three control questions. After all subjects had answered correctly the control questions, subjects played the principal-agent game once. At the end of the one-shot interaction, subjects' payoffs were displayed on their screens. In the four repetitions, ECUs were converted to Euro in the calculation of subjects' final earnings at a conversion rate of 10 ECUs to 1 Euro. Average earnings per subject are shown in Table 1.

Before leaving the laboratory, subjects were asked to complete a questionnaire in which they had to state their work motivation in five hypothetical scenarios (subjects did not know at the beginning of the session that they would have to answer this questionnaire). Different subjects answered different versions of the questionnaire, the two versions being identical to the ones $F \& \mathrm{~K}$ administered in their questionnaire study. For each scenario, there is a condition in which the principal trusts the agent and a condition in which the principal controls the agent. Each subject went through only one of the two conditions for a given scenario (see Appendices C and D of the electronic supplementary material). Answering the questionnaire was mandatory but not incentivized and subjects were informed that their answers to the questionnaire would not affect their previous earnings. In Experiments 2, 3, 4 and 5, once all subjects had answered the questionnaire they were asked to report their year of birth, their gender, and their academic major. Finally, subjects privately retrieved their earnings (including the show-up fee in the four repetitions).

\section{Results}

In this section, we first present our main findings concerning the behavior of agents and principals in the four repetitions. Subjects' answers to the questionnaire are discussed next. Third, we examine the influence of hypothetical incentives on the play 
of the principal-agent game. Finally, we analyze in detail the differences in agents' behavior between our samples and F\&K's sample.

We report the results of two-sided statistical tests throughout the paper and acceptance or rejection of the null hypothesis is always based on a 5 percent level of significance. From now on, we refer to the two principal's choices as the control and no-control conditions and the term "significance" is intended in its statistical rather than substantive sense.

\subsection{The four repetitions}

Our first result concerns the hidden costs of control and is qualitatively in line with F\&K's first result in Experiments 1, 3 and 5 but not in Experiment 2.

Result 1 We observe significant hidden costs of control in Experiments 1, 3 and 5. Hidden costs of control are insignificant in Experiment 2.

Support We follow F\&K's procedure and modify the distribution of transfers in the no-control condition such that any transfer strictly lower than $\underline{x}$ is set equal to $\underline{x}$. In Experiments 1, 3 and 5, we reject the null hypothesis that the modified distribution in the no-control condition is the same as the distribution in the control condition (exact Wilcoxon signed rank tests for paired observations: $p$-value $<0.01$ and $p$-value $=$ 0.016 in Experiment 1's C5 and C10 treatments respectively; p-values $<0.01$ in Experiments 3 and 5). In Experiment 2, we never reject the null hypothesis that the modified distribution in the no-control condition is the same as the distribution in the control condition (exact Wilcoxon signed rank tests for paired observations, $p$-value $=0.177$ in $\mathrm{C} 5$ and $p$-value $=0.068$ in $\mathrm{C} 10$ ).

Though hidden costs of control are not significant in all our samples, we confirm F\&K's experimental finding that they are common. Still, hidden costs of control should be taken seriously only if they are substantial enough to undermine the effectiveness of economic incentives. ${ }^{7}$ Our second result establishes that in almost none of our control treatments do the hidden costs significantly outweigh the benefits of control. This result contradicts F\&K's second result and is the key contribution of our paper. $^{8}$

\footnotetext{
${ }^{7}$ As already mentioned, for a given agent's pair of transfers $\left(x^{C}, x^{N C}\right)$, the principal's decision to control entails hidden costs if $d=x^{C}-\max \left\{\underline{x}, x^{N C}\right\}<0$, i.e. the agent's indirect choice shift due to control is negative. According to F\&K's procedure, for a given sample of agents' choices, we observe hidden costs of control if the sum of the ranks of the negative $d$ 's is sufficiently larger than the sum of the ranks of the positive $d$ 's. Hidden costs of control can therefore be observed in samples which comprise mostly selfish agents since the choices of such agents are not taken into account by the procedure.

${ }^{8}$ It has been argued that students in Jena, which was part of the communist country East Germany, might be less bothered by the imposition of control than Zurich students because East Germany scores much lower on self-expression than Switzerland and a low self-expression score implies a relatively favorable attitude towards authority (the argument builds on Inglehart 2000). Our findings in Experiments 2 and 3 do not support the conjecture that self-expression positively correlates with control aversion in the laboratory because Italy scores higher on self-expression than East Germany (the self-expression dimension is extracted from the World Values Survey, a large investigation of attitudes, values and beliefs around the world; see http://www.worldvaluessurvey.org/).
} 
Result 2 In all treatments except Experiment 3's low control treatment, hidden costs do not significantly outweigh benefits of control. In Experiment 2's medium control treatment, benefits of control significantly outweigh hidden costs of control.

Support Table 2 shows agents' transfers as a function of the principal's decision in treatments $\mathrm{C} 5$ and $\mathrm{C} 10$. In each of the five panels, the first row reports average transfers for each condition followed by the average difference between transfers in the two conditions. The second row reports for each condition the standard deviation followed by 1 st quartile followed by median followed by 3rd quartile, and the $95 \%$ bootstrapped confidence interval for the average difference (based on $10^{5}$ replicates). In Experiment 3's C5 treatment, both the average and the median number of ECUs transferred by the agents to the principals are higher in the no-control condition than in the control condition and significantly so (exact Wilcoxon signed rank test for paired observations, $p$-value $<0.01) .{ }^{9}$ In Experiment 1's C5 treatment, Experiment 3's C10 treatment, and Experiment 5's C5 and C10 treatments, both the average and the median number of ECUs transferred by the agents to the principals are higher in the no-control condition than in the control condition but not significantly so (exact Wilcoxon signed rank tests for paired observations, $p$-value $=0.106$, $p$-value $=0.3026$, $p$-value $=0.548$ and $p$-value $=0.985$ respectively $)$. In Experiment 1's C10 treatment and Experiment 2's C5 treatment, both the average and the median number of ECUs transferred by the agents to the principals are (weakly) higher in the control condition than in the no-control condition but not significantly so (exact Wilcoxon signed rank tests for paired observations, $p$-value $=0.307$ and $p$-value $=0.720$ respectively). ${ }^{10}$ In Experiment 2's C10 treatment, both the average and the median number of ECUs transferred by the agents to the principals are higher in the control condition than in the no-control condition and significantly so (exact Wilcoxon signed rank test for paired observations, $p$-value $=0.046$ ). Note that in all control treatments the variance of transfers is higher in the no-control condition than in the control condition. Finally, with the help of exact Kruskal-Wallis tests, we look for differences in our four samples and F\&K's sample in terms of agents' transfers in the control and no-control condition separately. We never reject the null hypothesis that agents' transfers in the control condition are identical in all five samples ( $p$-value $=0.429$ in C5 and $p$-value $=0.563$ in C10). On the other hand, we always reject the null hypothesis that agents' transfers in the no-control condition are identical in all five samples ( $p$-value $<0.01$ in $\mathrm{C} 5$ and $\mathrm{C} 10)$.

An interesting aspect of F\&K's experimental design is that agents' heterogeneity in the behavioral reaction to control can be analyzed. The use of the strategy method permits to quantify the number of agents who react positively, neutrally, or negatively to the principal's implementation of control. Result 3 summarizes our findings

\footnotetext{
${ }^{9}$ Unlike the procedure employed to obtain Result 1, no transformation is applied to the distribution of transfers in the no-control condition to obtain Result 2.

${ }^{10}$ In each control treatment, hidden costs do not significantly outweigh benefits of control for a sample which combines the samples of Experiments 1 and 5 (exact Wilcoxon signed rank tests for paired observations, $p$-value $=0.113$ in $\mathrm{C} 5$ and $p$-value $=0.471$ in $\mathrm{C} 10)$.
} 


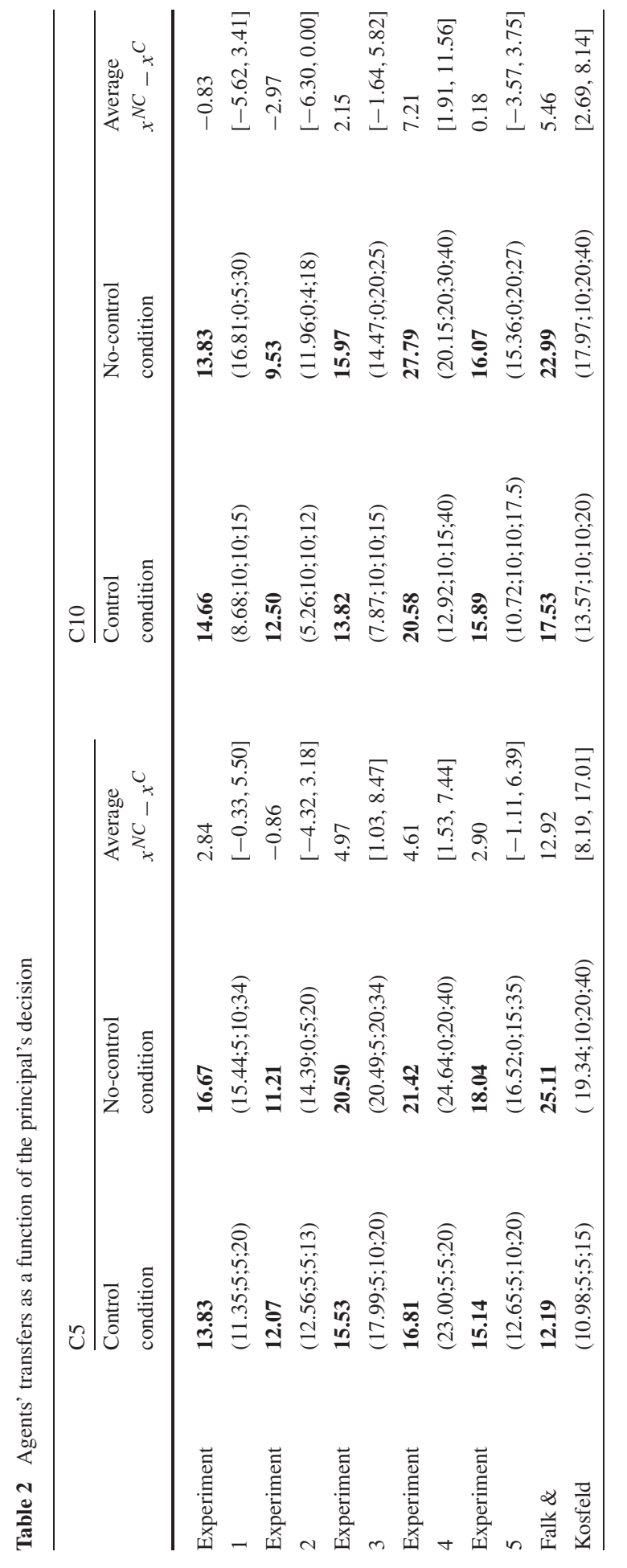


about the heterogeneity of agents' behavioral reaction to control. Negative reactions to the implementation of control are far less prevalent than in F\&K's C5 and C10 treatments.

Result 3 There is heterogeneity among the agents with regard to their behavioral reaction to control. In Experiment 2, the majority of agents always reacts positively to control. In Experiment 5, the largest group of agents always reacts positively to control. In Experiment 1, the reaction to control by the largest group of agents in C5 and $\mathrm{C} 10$ is respectively negative and positive. In Experiment 3, the largest group of agents always reacts negatively to control.

Support Table 3 summarizes the agents' behavioral reaction to control in the different samples. The relative share of agents who react negatively is much lower in our four samples (at most $40.62 \%$ and $45.45 \%$ in C5 and C10 respectively) than in F\&K's sample where negative reactions are the clear majority $(64.29 \%$ and $56.94 \%$ in C5 and $\mathrm{C} 10$ respectively). On the other hand, the relative share of agents who react positively is higher in our four samples (at least $23.33 \%$ and $39.40 \%$ in C5 and $\mathrm{C} 10$ respectively) than in F\&K's sample (20.00\% and $25.00 \%$ in C5 and C10 respectively). In both control treatments, Fisher's exact tests reject the null hypothesis that the frequencies of the different reactions to control-henceforth reaction frequencies-are identical in F\&K's sample and in Experiment 1 and 2's samples ( $p$-value $=0.046$ in Experiment 1's C5 treatment and $p$-value $<0.01$ otherwise). Similarly, a Fisher's exact test rejects the null hypothesis that in the low control treatment reaction frequencies are identical in F\&K's and Experiment 5's samples ( $p$-value $=0.033$ ). However, Fisher's exact tests cannot reject the null hypothesis that reaction frequencies in F\&K's sample are identical to those in Experiment 5's C10 treatment ( $p$-value $=0.077)$ and in both control treatments of Experiment 3 ( $p$-value $=0.054$ in $\mathrm{C} 5$ and $p$-value $=0.337$ in $\mathrm{C} 10$ ). It is important to emphasize that the difference in the fraction of negative reactions to control observed by $\mathrm{F} \& \mathrm{~K}$ and the one we observe is only partly responsible for the difference in the magnitude of hidden costs of control. In all low control treatments, agents who react negatively in F\&K's sample exhibit a much stronger negative response to control than in our samples (e.g. more than twice the response observed in Experiment 1 or 2). In medium control treatments, negative responses to control are comparable except in Experiment 2 where they are lower.

Like in F\&K's sample, principals' behavior in our samples is in line with the hypothesis that most principals took a profit-maximizing decision anticipating quite well the distributions of conditional transfers. ${ }^{11}$ Since hidden costs of control of lower magnitude are observed in our samples compared to F\&K's sample, different conclusions are derived from the same hypothesis as summarized in our fourth result.

Result 4 In all treatments except Experiment 5's C5 treatment, at least half the principals chooses to control. The proportion of principals who control is signifi-

\footnotetext{
${ }^{11}$ After having made their decision, principals were asked to state their expectation about the number of ECUs transferred by the agent (expectations were not incentivized). Details about principals' expectations can be obtained from the authors upon request.
} 
Table 3 Agents' behavioral reaction to control

\begin{tabular}{|c|c|c|c|c|c|c|}
\hline & \multicolumn{3}{|l|}{$\mathrm{C} 5$} & \multicolumn{3}{|l|}{$\mathrm{C} 10$} \\
\hline & Positive & Neutral & Negative & Positive & Neutral & Negative \\
\hline \multicolumn{7}{|l|}{ Experiment 1} \\
\hline Relative share (\%) & 23.33 & 36.67 & 40.00 & 58.62 & 13.79 & 27.59 \\
\hline Mean control transfer & 5.00 & 16.82 & 16.25 & 10.35 & 28.75 & 16.75 \\
\hline Mean no-control transfer & 0.00 & 16.82 & 26.25 & 1.18 & 28.75 & 33.25 \\
\hline \multicolumn{7}{|l|}{ Experiment 2} \\
\hline Relative share (\%) & 50.00 & 21.43 & 28.57 & 60.00 & 16.67 & 23.33 \\
\hline Mean control transfer & 8.00 & 19.17 & 13.88 & 10.33 & 14.80 & 16.43 \\
\hline Mean no-control transfer & 0.64 & 19.17 & 23.75 & 1.39 & 14.80 & 26.71 \\
\hline \multicolumn{7}{|l|}{ Experiment 3} \\
\hline Relative share (\%) & 25.00 & 34.38 & 40.62 & 39.40 & 15.15 & 45.45 \\
\hline Mean control transfer & 5.62 & 23.64 & 14.77 & 10.00 & 22.40 & 14.27 \\
\hline Mean no-control transfer & 0.62 & 23.64 & 30.08 & 0.77 & 22.40 & 27.00 \\
\hline \multicolumn{7}{|l|}{ Experiment 4} \\
\hline Relative share (\%) & 33.33 & 22.81 & 43.86 & 21.06 & 33.33 & 45.61 \\
\hline Mean control transfer & 5.84 & 42.69 & 11.68 & 11.25 & 36.32 & 13.38 \\
\hline Mean no-control transfer & 0.84 & 42.69 & 26.00 & 0.42 & 36.32 & 34.19 \\
\hline \multicolumn{7}{|l|}{ Experiment 5} \\
\hline Relative share (\%) & 39.29 & 25.00 & 35.71 & 42.86 & 25.00 & 32.14 \\
\hline Mean control transfer & 6.36 & 31.43 & 13.40 & 10.00 & 30.71 & 12.22 \\
\hline Mean no-control transfer & 1.36 & 31.43 & 27.00 & 0.83 & 30.71 & 25.00 \\
\hline \multicolumn{7}{|l|}{ Falk \& Kosfeld } \\
\hline Relative share (\%) & 20.00 & 15.71 & 64.29 & 25.00 & 18.06 & 56.94 \\
\hline Mean control transfer & 10.21 & 22.27 & 10.33 & 11.11 & 22.69 & 18.71 \\
\hline Mean no-control transfer & 4.79 & 22.27 & 32.13 & 1.94 & 22.69 & 32.32 \\
\hline
\end{tabular}

cantly higher than the proportion of principals who leave transfers unrestricted in the medium control treatments of Experiments 1 and 2 but not in the low control treatments or in Experiments 3's and 5's medium control treatment.

Support In Experiment 1 (Experiment 2, Experiment 3 \& Experiment 5 respectively), $50 \%$ and $83 \%$ (64\% and $77 \%, 53 \%$ and $64 \% \& 46 \%$ and $57 \%$ respectively) of our principals force the agent to transfer at least $\underline{x}$ ECUs in C5 and C10 respectively. Judging by binomial tests, the proportion of principals who leave transfers unrestricted is not significantly lower than the proportion of principals who control in the low control treatments ( $p$-value $=0.185, p$-value $=0.860$ and $p$-value $=0.851$ in Experiment 2, 3 and 5 respectively) as well as in the medium control treatments of Experiment 3 and 5 ( $p$-value $=0.163$ and $p$-value $=0.572$ respectively). In F\&K's C5 and C10 treatments, respectively $26 \%$ and $29 \%$ of the principals choose to control 
which is the least rewarding decision since average transfers are respectively $106 \%$ and $31 \%$ higher in the no-control condition.

\subsection{Questionnaires}

With the help of a questionnaire in which they presented 403 subjects with vignettes, F\&K studied how control and explicit incentives affect motivation in typical work environments. They constructed five different workplace scenarios, and, for each scenario, they presented a condition where the principal trusts the agent and a condition where the principal controls or uses explicit incentive devices. Each subject went through all five scenarios but faced only one of the two conditions for a given scenario. Based on a sample of 2015 responses, F\&K observe that, in all scenarios, stated work motivation is significantly lower when the principal controls than when he trusts the agent.

Like in F\&K's questionnaire study, our subjects had to indicate their work motivation on a five-level scale ranging from "very low" to "very high" for each scenario. The sample of 1820 subjects' answers from our first three repetitions is similar to F\&K's sample: in each scenario, the cumulative frequencies of subjects indicating that they have at most a medium motivation are always higher in the control than in the trust condition (for details see Appendix E of the electronic supplementary material). Like F\&K we observe that in all scenarios stated work motivation is significantly lower when the principal controls than when he trusts the agent (exact Wilcoxon signed rank tests, $p$-value $<0.01$ in each scenario). ${ }^{12}$ Even when restricting the sample to Trento subjects, for whom the impact of control is positive, we observe that in all scenarios except the fifth one stated work motivation is significantly lower in the control than in the trust condition (exact Wilcoxon signed rank tests, $p$-value $=0.278$ in scenario 5 and $p$-value $<0.01$ for all remaining scenarios).

Little revealed control aversion is inferred from the game play data of our first three repetitions but stated work motivation is substantially affected by the principal's monitoring policy according to our questionnaire data. This concomitance of dissimilar game play data and similar questionnaire data with respect to F\&K led us to conjecture that hypothetical incentives are conducive to substantial hidden costs of control in our samples. We now report a test of this conjecture. ${ }^{13}$

\subsection{The extension}

Result 5 summarizes our findings concerning agents' behavior in Experiment 4 which are qualitatively in line with $\mathrm{F} \& \mathrm{~K}$ 's results 1 to $3 .{ }^{14}$

\footnotetext{
${ }^{12}$ None of our conclusions changes if we include the 560 answers of Experiment 5.

${ }^{13}$ Alternatively, the implemented principal-agent game might not incorporate essential features of employment relationships which generate strong negative reactions to control in our samples.

${ }^{14}$ In Experiment 4, only $42 \%$ and $44 \%$ of the principals force the agent to transfer at least $x$ ECUs in $\mathrm{C} 5$ and $\mathrm{C} 10$ respectively. Judging by binomial tests, the proportion of principals who leave transfers unrestricted is not significantly higher than the proportion of principals who control ( $p$-value $=0.289$ in C5 and $p$-value $=0.427$ in $\mathrm{C} 10)$.
} 
Result 5 We observe significant hidden costs that significantly outweigh the benefits of control. The largest group of agents reacts negatively to the implementation of control.

Support Agents' transfers in Experiment 4 are summarized in the fourth panel of Table 2. We always reject the null hypothesis that the modified distribution in the no-control condition is the same as the distribution in the control condition (exact Wilcoxon signed rank tests for paired observations, $p$-value $<0.01$ in both treatments). Both the average and the median number of ECUs transferred by the agents to the principals are always higher in the no-control condition than in the control condition and significantly so (exact Wilcoxon signed rank tests for paired observations, $p$-value $\leq 0.01$ in both treatments). As shown in the fourth panel of Table 3 , the largest group of agents reacts negatively to the principal's implementation of control (43.86\% and $45.61 \%$ in C5 and C10 respectively). We never reject the null hypothesis that reaction frequencies are comparable in Experiment 4 and in F\&K (Fisher's exact test, $p$-value $=0.073$ in $\mathrm{C} 5$ and $p$-value $=0.151$ in $\mathrm{C} 10$ ).

\subsection{Differences between F\&K's sample and our samples}

The evidence collected in F\&K's principal-agent game shows that the magnitude of hidden costs of control and whether they significantly outweigh the benefits of control or not is determined by three factors: (i) The relative share of positive reactions to control (in any sample the average difference between transfers among such reactions roughly equals $-\underline{x}$ ); (ii) The relative share of negative reactions to control; and (iii) The strength of the negative reactions to control. With the help of five statistical models and a series of hypotheses tests, we now assess the relative importance of each of the three factors which ultimately enables us to pin down the source of the differences in agents' behavior between our samples and F\&K's sample.

Table 4 reports the estimates of two linear and three logistic regressions based on samples omitting agents who transfer more than 40 ECUs in at least one of the two control conditions. In each regression, the explanatory variables are the 12 experimental conditions (two control treatments in each of our five experiments and in F\&K's experiment). In Model 1, the dependent variable is the difference between transfers $x^{N C}-x^{C}$. The estimation method is OLS and we adjust standard errors for heteroscedasticity and small-sample bias with the help of the residual-variance estimator HC3 (McKinnon and White 1985). In Model 5, the same analysis is conducted on the subsample of agents who react negatively to control. In Model 2 (Model 3 and Model 4 respectively), the dependent variable is a dummy which takes value 1 if the reaction to control is positive (neutral and negative respectively) and 0 otherwise. Based on Logit estimates, we report for each experimental condition the predicted probability of falling into one of the three mutually exclusive categories of reaction to control. Finally, the lower panel of Table 4 reports Wald tests of the equality of the coefficients of F\&K C $\underline{x}$ and Expi $\underline{\mathrm{C}} \underline{x}$ with $\underline{x} \in\{5,10\}$ and $i \in\{1,2,3,4,5\} .{ }^{15}$

\footnotetext{
${ }^{15}$ Appendix F of the electronic supplementary material nicely complements Table 4.
} 
Table 4 Regressions on differences between transfers and reactions to control

\begin{tabular}{|c|c|c|c|c|c|c|c|}
\hline & & & & & eactions to $\mathrm{c}$ & ntrol & \\
\hline & & & $\begin{array}{l}x^{N C}-x^{C} \\
(1)\end{array}$ & $\begin{array}{l}\text { Positive } \\
\text { (2) }\end{array}$ & $\begin{array}{l}\text { Neutral } \\
\text { (3) }\end{array}$ & $\begin{array}{l}\text { Negative } \\
\text { (4) }\end{array}$ & $\begin{array}{l}\text { If } x^{N C}>x^{C}, \\
x^{N C}-x^{C}\end{array}$ \\
\hline & & $F \& K$ C5 & $10.52^{*}$ & $0.21^{*}$ & $0.16^{*}$ & $0.63^{*}$ & $18.60^{*}$ \\
\hline & & $\operatorname{Exp} 1 \mathrm{C} 5$ & 2.83 & $0.23^{*}$ & 0.37 & 0.40 & $10.00^{*}$ \\
\hline & & $\operatorname{Exp} 2 \mathrm{C} 5$ & -0.86 & 0.50 & $0.21^{*}$ & $0.29^{*}$ & $9.88^{*}$ \\
\hline & & Exp3 C5 & $5.13^{*}$ & $0.26^{*}$ & 0.32 & 0.42 & $15.31^{*}$ \\
\hline & & $\operatorname{Exp} 4 \mathrm{C} 5$ & $4.78^{*}$ & $0.35^{*}$ & $0.20^{*}$ & 0.45 & $14.32^{*}$ \\
\hline & & $\operatorname{Exp} 5 \mathrm{C} 5$ & 1.89 & 0.41 & $0.26^{*}$ & 0.33 & $11.78^{*}$ \\
\hline & & $F \& K \mathrm{C} 10$ & $5.26^{*}$ & $0.26^{*}$ & $0.19^{*}$ & 0.55 & $13.89^{*}$ \\
\hline & & $\operatorname{Exp} 1 \mathrm{C} 10$ & -0.83 & 0.59 & $0.14^{*}$ & $0.28^{*}$ & $16.50^{*}$ \\
\hline & & $\operatorname{Exp} 2 \mathrm{C} 10$ & -2.97 & 0.60 & $0.17^{*}$ & $0.23^{*}$ & $10.29^{*}$ \\
\hline & & Exp3 C10 & 2.15 & 0.39 & $0.15^{*}$ & 0.45 & $12.73^{*}$ \\
\hline & & Exp4 C10 & $4.75^{*}$ & $0.22^{*}$ & $0.35^{*}$ & 0.44 & $16.29^{*}$ \\
\hline & & Exp5 C10 & 0.18 & 0.43 & $0.25^{*}$ & 0.32 & $12.78^{*}$ \\
\hline & & $R$-squared & 0.179 & - & - & - & 0.734 \\
\hline & & Log likelihood & - & -291.014 & -250.421 & -316.213 & - \\
\hline & & Observations & 482 & 482 & 482 & 482 & 210 \\
\hline$\overline{\mathrm{H}}$ & & $F \& K \mathrm{C} 5=\operatorname{Exp} 1 \mathrm{C} 5$ & $p<0.01$ & $p>0.10$ & $p=0.03$ & $p=0.04$ & $p<0.01$ \\
\hline $\mathrm{Y}$ & & $F \& K \mathrm{C} 5=\operatorname{Exp} 2 \mathrm{C} 5$ & $p<0.01$ & $p<0.01$ & $p>0.10$ & $p<0.01$ & $p<0.01$ \\
\hline $\mathrm{P}$ & $\mathrm{T}$ & $F \& K \mathrm{C} 5=\operatorname{Exp} 3 \mathrm{C} 5$ & $p=0.04$ & $p>0.10$ & $p=0.08$ & $p=0.06$ & $p>0.10$ \\
\hline $\mathrm{O}$ & $\mathrm{E}$ & $F \& K \mathrm{C} 5=\operatorname{Exp} 4 \mathrm{C} 5$ & $p=0.01$ & $p=0.09$ & $p>0.10$ & $p=0.06$ & $p>0.10$ \\
\hline $\mathrm{T}$ & $\mathrm{S}$ & $F \& K \mathrm{C} 5=\operatorname{Exp} 5 \mathrm{C} 5$ & $p<0.01$ & $p>0.05$ & $p>0.10$ & $p=0.01$ & $p=0.07$ \\
\hline $\mathrm{H}$ & $\mathrm{T}$ & $F \& K \mathrm{C} 10=\operatorname{Exp} 1 \mathrm{C} 10$ & $p=0.03$ & $p<0.01$ & $p>0.10$ & $p=0.01$ & $p>0.10$ \\
\hline E & I & $F \& K \mathrm{C} 10=\operatorname{Exp} 2 \mathrm{C} 10$ & $p<0.01$ & $p<0.01$ & $p>0.10$ & $p<0.01$ & $p>0.10$ \\
\hline $\mathrm{S}$ & $\mathrm{N}$ & $F \& K \mathrm{C} 10=\operatorname{Exp} 3 \mathrm{C} 10$ & $p>0.10$ & $p>0.10$ & $p>0.10$ & $p>0.10$ & $p>0.10$ \\
\hline I & G & $F \& K \mathrm{C} 10=\operatorname{Exp} 4 \mathrm{C} 10$ & $p>0.10$ & $p>0.10$ & $p<0.05$ & $p>0.10$ & $p>0.10$ \\
\hline S & & $F \& K \mathrm{C} 10=\operatorname{Exp} 5 \mathrm{C} 10$ & $p=0.04$ & $p>0.10$ & $p>0.10$ & $p=0.04$ & $p>0.10$ \\
\hline
\end{tabular}

Notes: ${ }^{*}$ Significant at the 5 percent level.

In hypothesis testing, $p<0.05$ or $p>0.05$ means different from $5 \%$ at the third decimal place

We observe that: (i) In both control treatments, the differences between transfers are significantly smaller in Experiments 1 and 5 than in F\&K. These smaller differences are mainly due to significantly lower fractions of negative reactions to control. Significantly more positive reactions to control are observed in Exp1 C10 than in $\mathrm{F} \& \mathrm{~K} \mathrm{C10}$. The strength of negative reactions to control is significantly lower in Exp1 C5 than in F\&K C5; (ii) In both control treatments, the differences between transfers are significantly smaller in Experiment 2 than in $F \& K$, and significantly more positive (respectively less negative) reactions to control are observed in Exp2 than in F\&K. The strength of negative reactions to control is significantly lower in Exp2 C5 than in F\&K C5; (iii) In treatment C5, the differences between transfers are significantly smaller in Experiments 3 and 4 than in F\&K. Fractions of negative/positive reactions 
to control and the strength of negative reactions to control never significantly differ between F\&K and Experiments 3 and 4. ${ }^{16}$

In a nutshell, offering discretion is never profitable in our two experiments with performance-contingent earnings and balanced academic majors (Experiments 1 and 5). Accordingly, these two Jena samples differ from F\&K's sample and the main determinant of this difference is the frequency of negative reactions to control. ${ }^{17}$ Offering discretion is also never profitable in our experiment conducted in Trento with performance-contingent earnings and unbalanced academic majors (Experiment 2). Determinants of the difference with F\&K's results are the frequency of negative reactions to control and the frequency of positive reactions to control. Finally, agents' behavior is rather similar in F\&K's sample and in two of our samples: the Jena sample with unbalanced academic majors (Experiment 3 ) and the Jena sample with hypothetical incentives (Experiment 4).

\section{Conclusion}

In assessing the reproducibility of F\&K's experimental findings, we have largely confirmed the existence of significant hidden costs of control (Experiments 1, 3 and 5) but we have also established that hidden costs of control are not significant in all samples (Experiment 2). Most importantly, we mainly observe hidden costs of control of low magnitude which lead to low-trust principal-agent relationships. Hidden costs of control are usually not substantial enough to significantly undermine the effectiveness of economic incentives in our four repetitions (all 8 treatments except Experiment 3's low control treatment). ${ }^{18}$ These results again support the view that offering discretion is rarely profitable in non-personal relationships and in the absence of complementarities with other attributes of the job. The evidence collected in Experiment 4 reconciles the conflicting results of our play and questionnaire data.

F\&K's principal-agent game is a good starting point for the development of a tool to measure control aversion and for studying the relative importance of explicit and implicit incentives, and their interaction effects. Future experimental studies should implement a repetitive trial environment to elicit a more reliable measure of control aversion, and laboratory experiments should be combined with field experiments employing heterogeneous populations within a given country as well as across countries. Finally, game play data should be complemented with survey data on a large

\footnotetext{
${ }^{16}$ In most cases, Fisher's exact tests lead to the same qualitative conclusions as the Wald tests of columns (2), (3) and (4). Exceptions are: Fisher's exact tests reject the null hypothesis that in C5 frequencies of negative reactions to control are identical in F\&K and Experiments 3 and 4 ( $p$-values $=0.03$ ). A Fisher's exact test rejects the null hypothesis that in $\mathrm{C} 5$ frequencies of neutral reactions to control are identical in $\mathrm{F} \& \mathrm{~K}$ and Experiment 3 ( $p$-value $=0.04)$. A Fisher's exact test does not reject the null hypothesis that in $\mathrm{C} 10$ frequencies of neutral reactions to control are identical in $\mathrm{F} \& \mathrm{~K}$ and Experiment 4 ( $p$-value $=0.06$ ).

${ }^{17}$ Compared to Experiment 1, the sample size of Experiment 5 is small given that subjects' decisions are likely to be noisier. A larger sample size would have increased our confidence in the robustness of Experiment 5's results. Unfortunately, recruiting more subjects in Experiment 5 was beyond our financial budget.

${ }^{18}$ Two replication and extension studies of F\&K's medium control treatment, Hagemann (2007) and Schnedler and Vadovic (2011), validate our experimental results in their replication treatments.
} 
spectrum of individual characteristics, subjective attitudes to control and information about the workplace environment.

Acknowledgements Experiments 1 to 4 were funded by the Max Planck Society. Experiment 5 was funded by the program ProChance of the University of Jena. We are indebted to Michael Kosfeld who provided us with the software source code, instructions and questionnaires of Falk and Kosfeld's (2006) study. We are grateful to Petra Hagemann, Andreas Ortmann, Wendelin Schnedler, audiences at IMPRS workshops and especially to Jürgen Bracht and Birendra Kumar Rai for helpful comments. Discussions with Urs Fischbacher were very supportive. Christian Adam, Sascha Bähr, Markus Heinemann, Adrian Liebtrau, and Karolin Schröter provided valuable research assistance. The comments of the Editor, Jacob Goeree, and two anonymous referees shaped the paper in important ways and we are grateful for the challenges they raised.

\section{References}

Antonakis, J., \& Atwater, L. (2002). Leader distance: A review and a proposed theory. Leadership Quarterly, 13(6), 673-704.

Barkema, H. G. (1995). Do top managers work harder when they are monitored? Kyklos, 48(1), 19-42.

Bartling, B., Fehr, E., \& Schmidt, K. M. (2011, forthcoming). Screening, competition, and job design: Economic origins of good jobs. American Economic Review.

Deci, E. L. (1971). Effects of externally mediated rewards on intrinsic motivation. Journal of Personality and Social Psychology, 18, 105-115.

Dickinson, D., \& Villeval, M. C. (2008). Does monitoring decrease work effort? The complementarity between agency and crowding-out theories. Games and Economic Behavior, 63, 56-76.

Dufwenberg, M., \& Kirchsteiger, G. (2004). A theory of sequential reciprocity. Games and Economic Behavior, 47(2), 268-298.

Ellingsen, T., \& Johannesson, M. (2008). Pride and prejudice: The human side of incentive theory. American Economic Review, 98(3), 990-1008.

Falk, A., \& Fischbacher, U. (2006). A theory of reciprocity. Games and Economic Behavior, 54(2), $293-$ 315 .

Falk, A., \& Kosfeld, M. (2006). The hidden costs of control. American Economic Review, 96(5), 16111630 .

Fischbacher, U. (2007). z-Tree: Zurich toolbox for ready-made economic experiments. Experimental Economics, 10(2), 171-178.

Frey, B. S. (1993). Does monitoring increase work effort? The rivalry between trust and loyalty. Economic Inquiry, 31, 663-670.

Greiner, B. (2004). An online recruitment system for economic experiments. In K. Kremer, V. Macho (Eds.) Forschung und wissenschaftliches Rechnen 2003. GWDG Bericht 63 (pp. 79-93). Ges. für Wiss. Datenverarbeitung, Göttingen.

Hagemann, P. (2007). What's in a frame: On demand effects and trust in experimental studies. Manuscript. University of Cologne.

Inglehart, R. (2000). Culture and democracy. In L. Harrison \& S. Huntington (Eds.), Culture matters: How values shape human progress. New York: Basic Books.

McKinnon, J. G., \& White, H. (1985). Some heteroskedasticity-consistent covariance matrix estimators with improved finite sample properties. Journal of Econometrics, 29, 305-325.

Rabin, M. (1993). Incorporating fairness into game theory and economics. American Economic Review, 83(5), 1281-1302.

Roth A. E. Prasnikar, V., Okuno-Fujiwara, M., \& Zamir, S. (1991). Bargaining and market behavior in Jerusalem, Ljubljana, Pittsburgh, and Tokyo: An experimental study. American Economic Review, 81(5), 1068-1095.

Schnedler, W., \& Vadovic, R. (2011, forthcoming). Legitimacy of control. Journal of Economics and Management Strategy 20.

von Siemens, F. (2011). Intention-based reciprocity and the hidden costs of control. Tinbergen Institute Discussion Paper 115/1.

Sliwka, D. (2007). Trust as a signal of a social norm and the hidden costs of incentive schemes. American Economic Review, 97(3), 999-1012.

Stanton, J. M. (2000). Reactions to employee performance monitoring: Framework, review, and research directions. Human Performance, 13(1), 85-113. 